\title{
Effect of the botanical compound LCS102 on innate immunity
}

\author{
ZOYA COHEN $^{1 *}$, YAIR MAIMON $^{1 *}$, RAANAN BERGER $^{2}$, \\ OFIR MORAG $^{2}$, TALIA GOLAN ${ }^{2}$ and NOAH SAMUELS ${ }^{3}$ \\ ${ }^{1}$ Tal Center for Integrative Medicine; ${ }^{2}$ Institute of Oncology, Sheba Medical Center, Ramat Gan 52621; \\ ${ }^{3}$ Sackler Faculty of Medicine, Tel Aviv University, Tel Aviv 69978, Israel
}

Received May 17, 2019; Accepted March 24, 2020

DOI: $10.3892 /$ br.2020.1323

\begin{abstract}
Innate immunity serves an important role in the healthy population, providing surveillance and protection against infections. Chemotherapy suppresses the body's immune system, including neutrophil and natural killer (NK) cell numbers and activity. This leads to an increased risk of infection which often requires the reduction or even discontinuation of the chemotherapeutic regimens. The botanical formula LCS102 was designed to stimulate the body's immune system. The effect of the formula on innate immunity was examined in human blood samples, as were its effect on the anti-cancer activity of chemotherapeutic agents on human cancer cells. Blood samples drawn from 20 volunteers (19 healthy subjects; 1 patient with breast cancer undergoing chemotherapy) and were exposed to LCS102. The effects on neutrophil and NK cell activity were tested using FACS. The anti-cancer effects of LCS102 were tested on T24, A549, MCF7, PANC-1 and U2OS human cancer cell lines, as were the effects of the formula on doxorubicin, Taxol, etoposide and cisplatin-treated cells using a sulforodamine B viability assay. LCS102 was shown to significantly increase the percentage of activated neutrophils and NK cells in the blood samples tested. The formula did not inhibit the cytotoxic effects of the chemotherapeutic agents, and in certain cases increased their anti-cancer activity. Further research is required to improve our understanding of the clinical value of LCS102; however, it may serve as an adjuvant during chemotherapy, to reduce the effects of chemotherapy on innate immunity.
\end{abstract}

\section{Introduction}

The body's innate immune system is mediated by granulocytes, dendritic cells, macrophages, natural killer (NK) and

Correspondence to: Dr Yair Maimon, Tal Center for Integrative Medicine, Institute of Oncology, Sheba Medical Center, 2 Derech Sheba, Ramat Gan 52621, Israel

E-mail: yair@tcm.org.il

${ }^{*}$ Contributed equally

Key words: botanical formula, LCS102, LCS101, innate immunity, neutrophils, natural killer cells mast cells, and serves an important role in protecting the body from infection. Innate immunity is considered the body's first line of defense against antigenic challenges, with imbalances in this aspect of the immune system increasing the risk of the development of cancer, as well as adversely affecting treatment outcomes and prognosis (1). Chronic inflammation leads to irreversible changes in tissue remodeling and induces oxidative stress, resulting in altered protein and DNA structure, and thus the eventual development of cancer (1). Neutropenia, a common adverse effect of chemotherapy, is frequently accompanied by fever (febrile neutropenia) which is assumed to be a manifestation of an underlying infection, requiring hospitalization and intravenous administration of antibiotics (2). A suppressed innate immune system resulting from a chemotherapeutic regimen may take a long time to recover, particularly for the recovery of NK cells, which serves an important role in chemotherapy with their potential for anti-tumor activity (3). Although conventional oncology provides treatment for chemotherapy-induced neutropenia, this does not address other components of the innate immune system, and is often accompanied by significant adverse effects (4).

Several herbal medicinal products have been shown to exhibit significant effects on the immune system, and may thus be used to improve the quality of life-associated outcomes in cancer patients undergoing chemotherapy (5-7). The botanical formula LCS101 was designed based on the principles of traditional Chinese medicine (TCM) for the treatment of patients undergoing chemotherapy for breast cancer. In a randomized, double-blind, placebo-controlled clinical trial, LCS101 was shown to significantly reduce anemia and neutropenia in 65 female patients with locally advanced breast cancer undergoing anthracycline and taxane-based treatment regimens (8). LCS101 was also shown to exhibit significant and selective anti-cancer effects, inducing necrosis and apoptosis in cancer cell lines, whilst protecting non-tumor cells from the cytotoxic effects of chemotherapy (9). Finally, LCS101 was shown to display several anti-cancer immune-modulating effects, including dose-dependent upregulation of NK cell activation and T-cell proliferation (10).

LCS102 is a botanical compound which is similar to LCS101 in that it is also based on the principles of TCM, although it is directed at treating the body's immune response during chemotherapy. The aim of the present study was to examine the effects of LCS102 on innate immunity, as well as testing its effect on chemotherapy. 


\section{Materials and methods}

Antibodies and reagents. Doxorubicin, Taxol, etoposide and cisplatin were acquired from Sigma Aldrich; Merck KGaA. Each chemotherapeutic agent was used in incrementally increased concentrations (from 0 to $3 \mathrm{mg} / \mathrm{ml}$ ) to $1 \mathrm{mg} / \mathrm{ml}$ of the LCS102 preparation (described in detail below) for 30 min each. RPMI-1640, L-glutamine, FBS, trypsin and PBS were purchased from Biological Industries. Red blood cell lysis buffer, Alexa Fluor 488-conjugated mouse anti-human CD56 IgG1к (cat. no. 318312; CD56-AF), PE-conjugated mouse anti-human CD69 IgG1к (cat. no. 310906; CD69-PE),

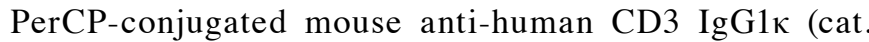
no. 344814 CD3-PerCP), PE-conjugated mouse IgG1 $\kappa$ MOPC-21 isotype control antibody, Alexa Fluor 488-conju-

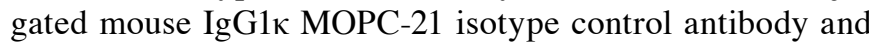
PerCP-conjugated mouse IgG1к MOPC-21 isotype control antibody were all purchased from BioLegend, Inc. IL-2 was from purchased PeproTech, Inc.

Donor blood samples. The present study was approved by the Sheba Medical Center Institutional Review Board (Ramat Gan, Israel). For the present study, 20 volunteers ( 12 male, 8 female) were asked to donate blood, 19 of these were healthy donors with no chronic medical illness or medication use, including drugs with a potential to alter the immune response; and 1 of the female patients had a carcinoma of the breast and was undergoing chemotherapy. All volunteers provided written informed consent, and $5 \mathrm{ml}$ of blood was drawn from each donor and collected in heparin-treated tubes (Greiner Bio-One, $\mathrm{GmbH}$ ). Each tube was inverted several times and then treated as described below.

Human cell lines. A549 (lung carcinoma), MCF7 (breast adenocarcinoma), PANC-1 (pancreatic epithelioid carcinoma), U-2 OS (osteosarcoma) and T24 (bladder transitional cell carcinoma) were purchased from American Type Culture Collection, and were authenticated using STR profiling. All cells were cultured in RPMI-1640 with $10 \%$ FBS, 2 mM L-glutamine, $100 \mu \mathrm{g} / \mathrm{ml}$ Pen/Strep (Biological Industries) in a $37^{\circ} \mathrm{C}$ humidified incubator with $5 \% \mathrm{CO}_{2}$.

Preparation of LCS102 and LCS101 botanical formulas. LCS102 is comprised of the following herbal components: Astragalus membranaceus, Poriae cocos, Atractylodes macrocephala, Ligustrum lucidum, Lycium chinense, Ganoderma lucidum and Cordyceps sinensis, extracts of which are manufactured in accordance with good manufacturing practice guidelines, and imported under license (BARA Herbs, Yokneam, Israel) in accordance with the regulations of the Israel Ministry of Health. All batches of the final product were analyzed and certified to be free of contaminants, such as heavy metals, microbial contamination, pesticide residues and mycotoxins. A dry extract powder of the LCS102 and LCS101 formulas (BARA Herbs Ltd) was dissolved in PBS at a concentration of $100 \mathrm{mg} / \mathrm{ml}$, and incubated for $30 \mathrm{~min}$ with heating and occasional vortexing. The solution was then centrifuged at $4,300 \mathrm{x}$ g for $5 \mathrm{~min}$, and the supernatant was filtered through a $0.45 \mu \mathrm{m}$ Millex PVDF filter (Merck KGaA). Solubility was estimated by cryophilization and weighing of the pellet, and was estimated to be $\sim 50 \%$.

Treatment, preparation and staining of blood samples. Blood samples $(100 \mu \mathrm{l})$ were plated in U-shaped 96 -well plates and treated in triplicate immediately with $0-3 \mathrm{mg} / \mathrm{ml}$ of the botanical extracts. After $48 \mathrm{~h}$ of incubation at $37^{\circ} \mathrm{C}$ in a $5 \% \mathrm{CO}_{2}$ incubator treated aliquots $(50 \mu \mathrm{l})$ were stained with $3 \mu \mathrm{l}$ 1:1:1 CD56-AF/CD69-PE/CD3-PerCP or an isotype-matched control antibody mix for $30 \mathrm{~min}$ at room temperature, lysed with red blood cell lysis buffer, washed twice with PBS and re-suspended in PBS for subsequent FACS analysis. In all experiments PBS-treated samples were used as a negative control, and an IL-2 $(20 \mathrm{U} / \mathrm{ml})$-treated sample (prepared at the same temperature and for the same duration as the botanical compounds) as the positive control.

FACS analysis. The samples were analyzed using a $\mathrm{BD}$ FACSCalibur flow cytometer (BD Biosciences), using a three-color protocol with appropriate compensations. The results were analyzed using WinMDI version 2.9 (Purdue University Cytometry Laboratories) using the following methodology: First, a forward scatter (FSC)/ side scatter (SSC) dot plot was used to select neutrophil (R1, blue) and lymphocyte (R2, green) populations (Fig. 1A). Lymphocytes were further resolved using a CD3-PerCP/CD56-AF488 logarithmic dot plot (Fig. 1B) to select CD3-/CD56 ${ }^{+} \mathrm{NK}$ cells (R4, purple) population. Finally, each population was analyzed for CD69 expression on a CD69-PE logarithmic histogram plot, with appropriate gating (neutrophils, Fig. 1C; NK cells, Fig. 1D). An activated population marker (M1) was set in accordance with the isotype control staining of each population (data not shown). Due to differences in self-fluorescence, the settings for the activation markers differ between NK cells and neutrophils.

Treatment of cancer cell lines and Sulforodamine B (SRB) viability assay. Sulforodamine B, trichloroacetic acid and acetic acid were purchased from Sigma Aldrich; Merck KGaA. A total of $3 \times 10^{3}$ cells were plated per/well in a 96-well plate and incubated overnight. Subsequently, cells were treated in triplicates as described above and cultured for $48 \mathrm{~h}$. A SRB viability test was performed as follows: Cells were fixed for $1 \mathrm{~h}$ on ice with $10 \%$ trichloroacetic acid (v/v in RPMI-1640), washed trice with double distilled water, and dried and stained for $1 \mathrm{~h}$ at room temperature with $0.057 \%$ sulforodamine B (w/v in $1 \%$ acetic acid). After staining, the plates were washed three times with $1 \%$ acetic acid and then dried, and $200 \mu 1$ $10 \mathrm{mM}$ Tris was added to each well to solubilize the SRB. The absorbance was measured at $570 \mathrm{~nm}$ using an ELISA reader (Biotek Instruments, Inc.). Each experiment was repeated at least three times.

Statistical analysis. A total of 10 study groups were assessed, each in triplicate: LCS102, controls, IL-2, AME, AMA, PCAO, LCH, LLU, GLU and CSI. A total of 45 between-group comparisons were performed, using a non-parametric Kruskal-Wallis test, with a post-hoc Dunn's test. The data were analyzed using SPSS version 25 (IBM Corp.). $\mathrm{P}<0.05$ was considered to indicate a statistically significant difference. 

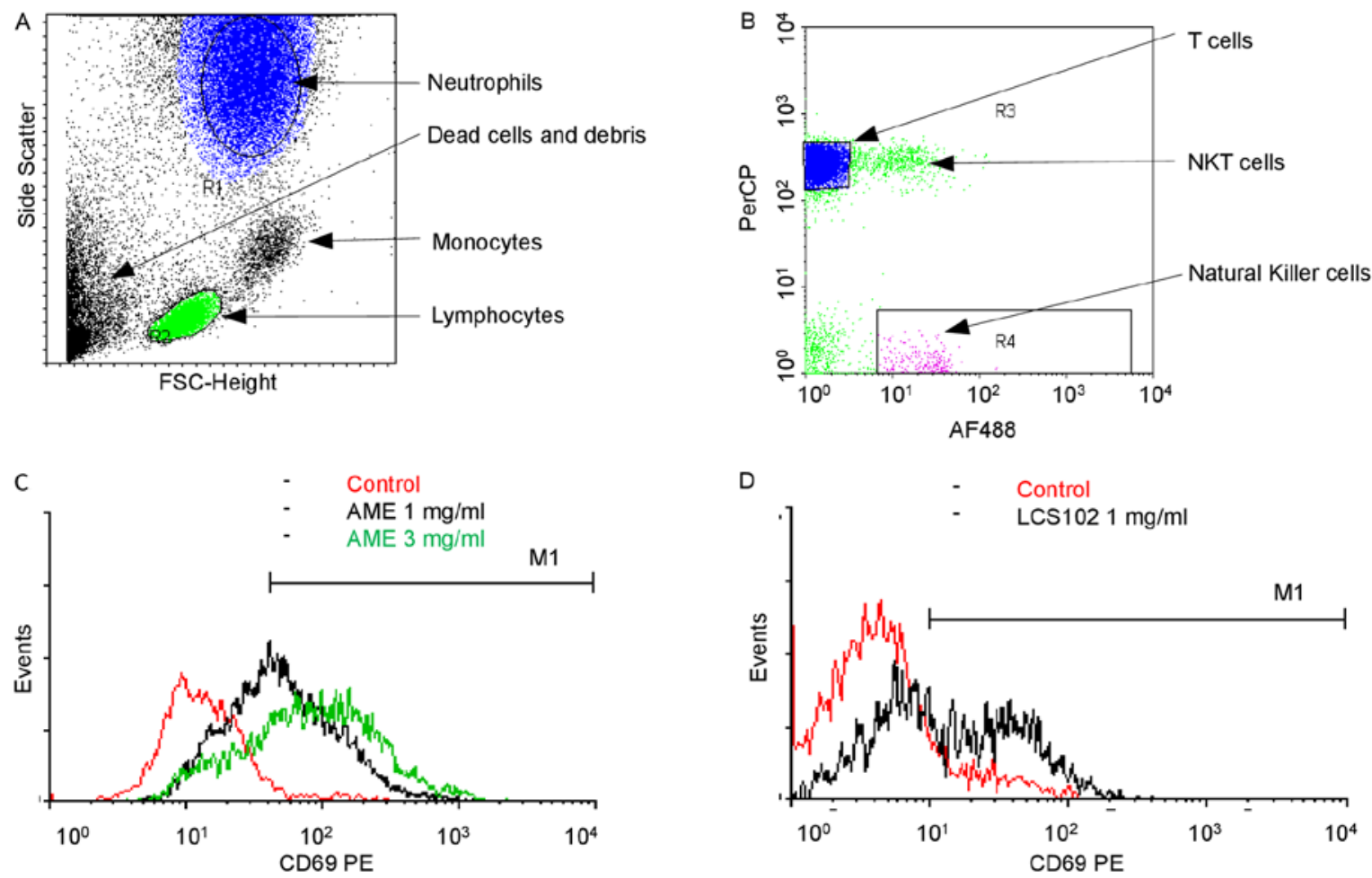

Figure 1. Selection and gating for the different cell populations. (A) Neutrophils (R1, blue) and lymphocytes (R2, green) populations were selected for using an FSC/SSC dot plot. (B) Lymphocyte populations were selected for on a CD3-PerCP/CD56-AF488 dot plot. CD3/CD56 ${ }^{+}$NK cells (R4, purple) population were selected. Samples were resolved on a CD69-PE histogram, gated and an activated population marker (M1) were selected for based on the isotype control staining for each population. (C) Histogram showing neutrophil activation in a blood sample from donor number 3 following treatment with Astragalus membranaceus extract. The M1 region contained the activated neutrophil population. (D) Histogram showing NK activation in a blood sample from donor number 1 treated with $1 \mathrm{mg} / \mathrm{ml}$ LCS102 extract. The M1 region shows the activated NK population. Due to differences in self-fluorescence, the activation markers differ for neutrophils in panel (C) and NK cells in panel (D). FSC, forward scatter; SSC, side scatter; NK, natural killer.
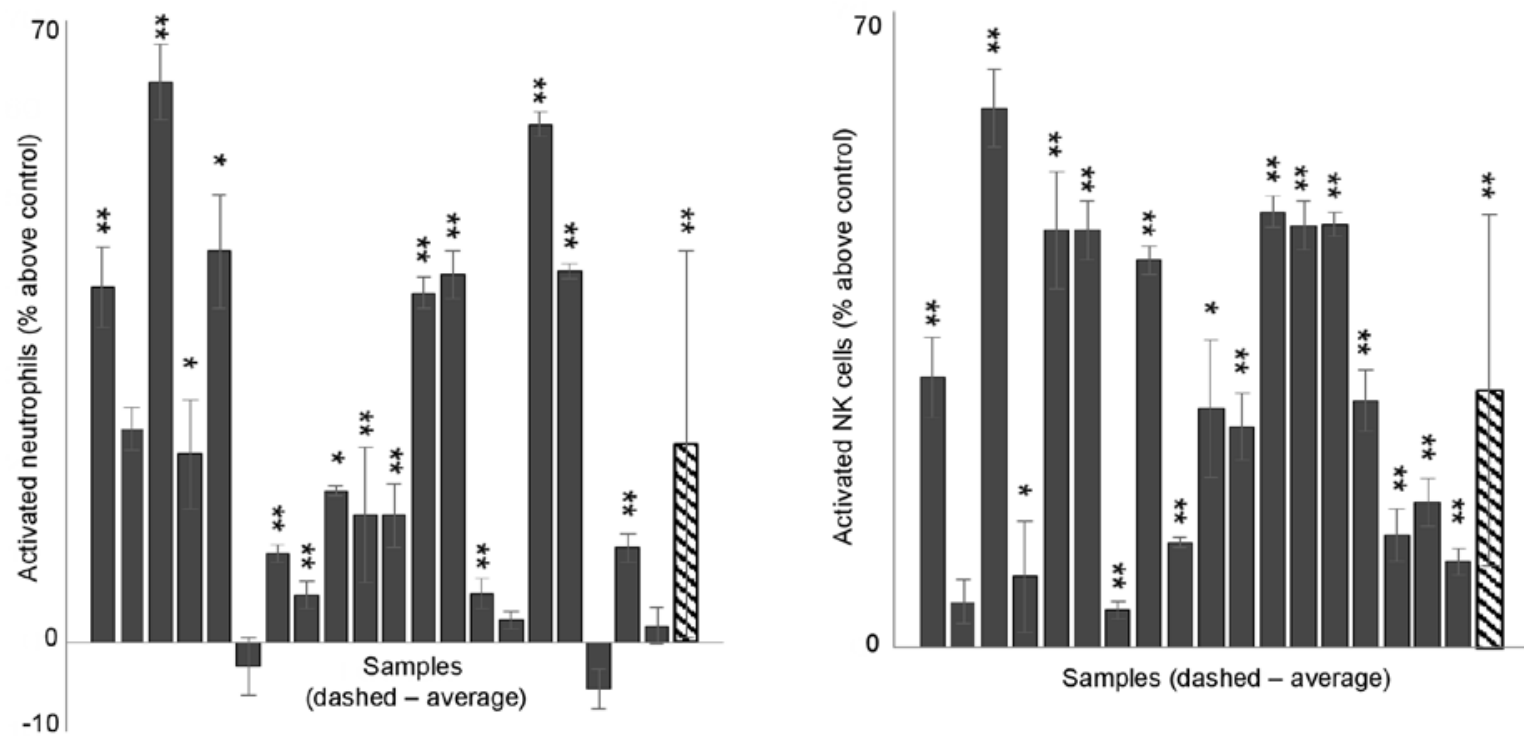

Figure 2. Activation of neutrophils and NK cells by LCS102. Blood samples were treated for $48 \mathrm{~h}$ with $1 \mathrm{mg} / \mathrm{ml}$ of LCS102, stained with CD3-PerCP/CD56-AF488/CD69-PE mix. The graphs show the percentage of activated cells above/below the negative control; filled columns show the values of individual blood samples and the patterned columns shows the average of all the samples. (A) Neutrophil activation. (B) $N K$ activation. ${ }^{*} \mathrm{P}<0.05,{ }^{* *} \mathrm{P}<0.01$ vs. negative control. NK, natural killer.

\section{Results}

LCS102 activates NK cells and neutrophils. The effects of the LCS102 formula on neutrophil and NK cell activation were examined in all of the blood samples. Samples were treated with $1 \mathrm{mg} / \mathrm{ml}$ of LCS102, PBS (negative control) or IL-2 (positive control) for $48 \mathrm{~h}$, stained with a CD3/CD56/CD69 fluorescent antibody mix and then analyzed using FACS. Neutrophil 


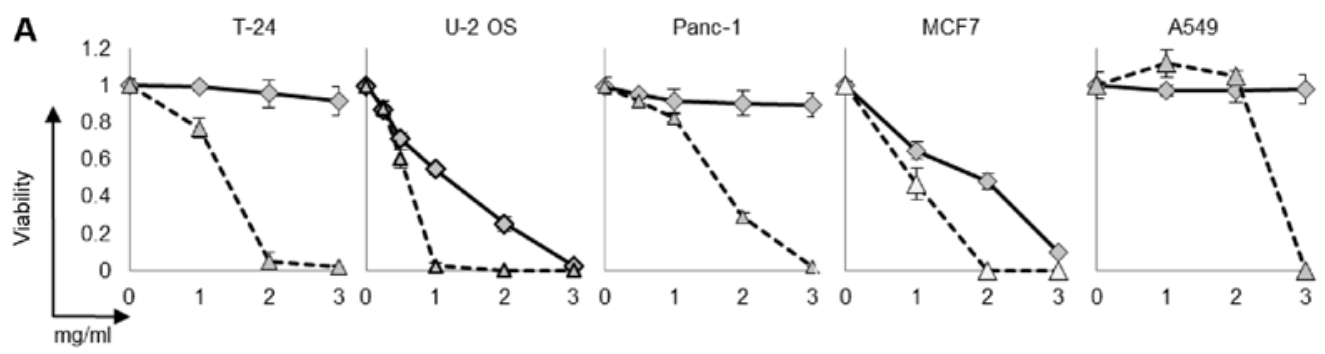

B
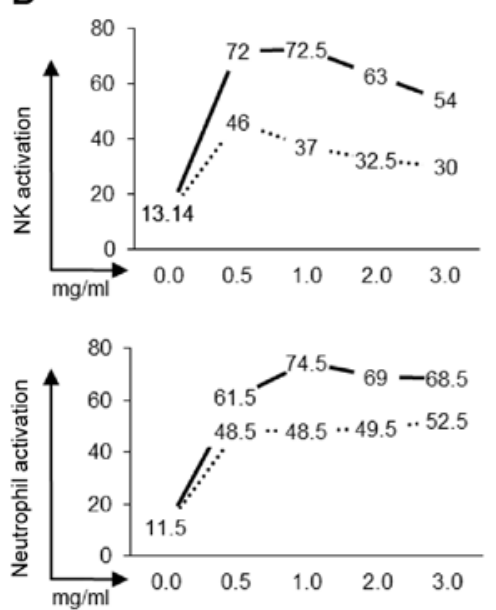
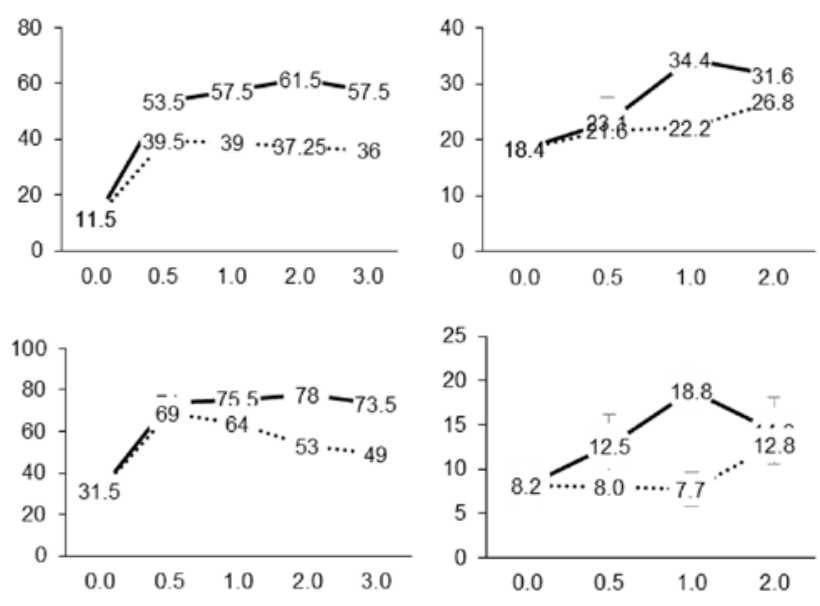

Figure 3. Comparison of anti-cancer and immunogenic effects of LCS102. (A) A549, T-24, Panc-1, MCF7 and U-2OS human cancer lines were treated for $48 \mathrm{~h}$ with indicated concentrations of LCS102 (continuous line) or LCS101 (dashed line), and the viability was assessed. (B) Blood samples from three healthy donors were treated for $48 \mathrm{~h}$ with increasing concentrations of LCS102 and LCS101 and analyzed as described in Fig. 1. NK, natural killer.

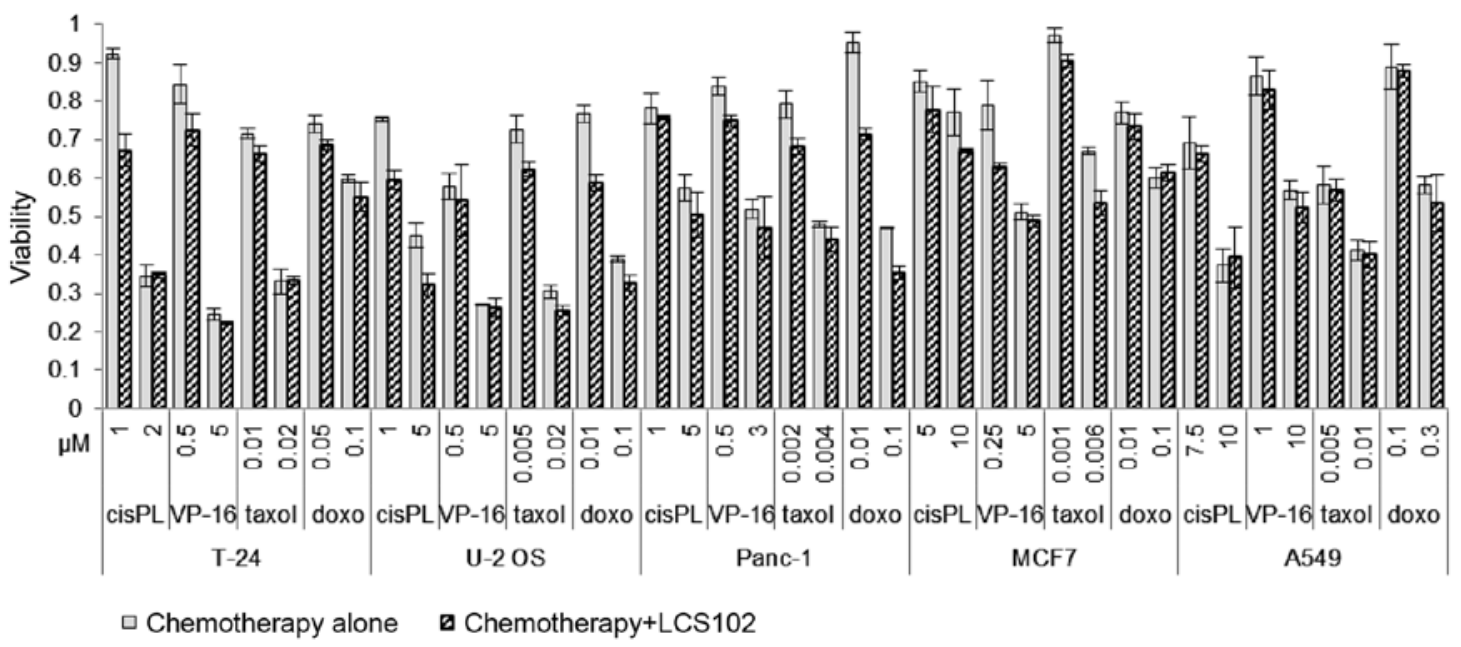

Figure 4. Cytotoxic effects of chemotherapeutic agents with and without LCS102. A549, T-24, Panc-1, MCF7 and U-2OS human cancer lines were treated for $48 \mathrm{~h}$ with the indicated concentrations of cisPL), etoposide (VP-16), Taxol and doxorubicin (doxo), either alone or with $1 \mathrm{mg} / \mathrm{ml}$ of LCS102, and the viability of cells was assessed. Data are presented relative to the respective untreated control cells. cisPL, cisplatin; VP-16, etoposide; doxo, doxorubicin.

populations were gated on an FSC/SSC dot-plot (Fig. 1A), and analyzed on a CD69-PE histogram (Fig. 1C). NK cells were first gated on an FSC/SSC dot-plot (Fig. 1A) for lymphocytes, and then on a CD3-PerCP/CD56-AF488 dot-plot for $\mathrm{CD}^{-} / \mathrm{CD}^{\circ} 6^{+}$(NK) cells (Fig. 1B), and analyzed on a CD69-PE histogram (Fig. 1D).

Fig. 2 shows the percentage of activated NK/neutrophils above/below the negative control value in each sample, as well as the average value of all samples for each treatment. LCS102 significantly elevated the percent of activated neutrophils and
NK cells in the majority of tested blood samples, although the extent of this activation varied among samples.

Comparing the cytotoxic and immune effects of LCS102 to LCS101. The effects of LCS102 on cancer cell survival were assessed and compared to with the LCS101 formula on a panel of five human cancer cell lines. As shown in Fig. 3A, LCS102 reduced the proliferation of two cancer cell lines, U-2 OS and MCF7, although it was less effective than the anti-cancer effects of LCS101. In order to address the effects of both formulas on 
NK and neutrophil activation, blood samples from three donors were treated with increasing ( 0 to $3 \mathrm{mg} / \mathrm{ml}$ ) concentrations of the compounds. As shown in Fig. 3B, LCS102 was considerably more effective in the activation of NK and neutrophils compared with LCS101.

LCS102 does not reduce the effectiveness of chemotherapy. Each cell line was exposed to incremental concentrations of cisplatin (cisPL), paclitaxel (Taxol), etoposide (VP-16) and doxorubicin (doxo). The addition of $1 \mathrm{mg} / \mathrm{ml}$ of LCS102 to each of the chemotherapy-treated cells did not result in reduced cytotoxic effects for any of the treatments in any of the cancer cell lines assessed. Additionally, in Panc-1 cells, the addition of LCS102 to doxorubicin resulted in a synergistic response, which manifested as increased cell death. This response was also observed with the addition of LCS102 to $1 \mu \mathrm{M}$ of cisplatin in T-24 cells (Fig. 4).

\section{Discussion}

In the present study, the botanical compound LCS102 was shown to exhibit a number of effects on innate immunity in blood samples taken from 20 subjects. The botanical formula was shown to significantly induce activation of neutrophils and NK cells in the majority of samples studied. Neutrophils are an important component of innate immunity, and neutropenia can impair the body's ability to respond to nascent infections, enabling bacteria and other organisms to multiply and invade the blood and organs (11). Neutropenia is a major complication of chemotherapy, and neutropenic fever and infections have long been a major cause of dose reduction and delays, with compromised treatment outcomes $(12,13)$. The use of granulocyte colony-stimulating factor is effective in reversing this complication, although this treatment is not without potential short- and long-term side effects, and is not cost-effective (4).

In previous studies, a number of LCS102 components have been shown to increase innate immunity, such as the herbs Astragalus membranaceous and Poriae cocus, which have been shown to increase macrophage and phagocyte activity $(14,15)$. The $\beta$-glycans of medicinal mushrooms such as Cordyceps sinensis have been shown to activate leucocytes, and stimulate phagocytic and cytotoxic activities (16). Herbal compounds have also been shown to increase NK cell activity, including LCS102. Astragalus membranaceus has been shown to stimulate NK-cells in human peripheral lymphocytes, as well as restore steroid-inhibited NK-cell activity (17). Polysaccharides of Astragalus can enhance NK cell activity in normal subjects, as well as in systemic lupus erythematosus patients (18). Finally, Cordyceps sinensis was shown to increase the activity of NK cells in cultured rat Kupffer cells (19).

When compared with LCS101, it was shown that LCS102 had a more significant effect on innate immunity. At the same time, the anti-cancer effects were less significant than those seen with LCS101. The present study strived to adhere to the TCM-based design of the two formulas; however, further research is required in order to verify and improve our understanding of the mechanism of each product.

In conclusion, the botanical compound LCS102 was shown to enhance the innate immune response in both healthy human blood samples, as well as in a sample from a patient with breast cancer undergoing chemotherapy. The variable response to the compounds, most pronounced with the effects on NK cell activity, should be further studied. Finally, the addition of LCS102 did not reduce the effect of chemotherapeutic drugs on cancer cells, and in some cell lines, increased the effects. However, the small size of the sample precludes reaching any conclusions regarding these findings. Further research is required to examine other aspects of LCS102, such as the biological effects of the formula and any potential interactions with chemotherapeutic agents, to improve our understanding of the potential clinical value of LCS102.

\section{Acknowledgements}

Not applicable.

\section{Funding}

No funding was received.

\section{Availability of data and materials}

The datasets used and/or analyzed during the present study are available from the corresponding author on reasonable request.

\section{Authors' contributions}

ZC, YM, RB, TG and NS designed the study. ZC, YM, OM and NS performed the experiments. ZC, YM, OM, TG, RB and NS analyzed the data. ZC, YM, OM, TG, RB and NS wrote the manuscript. All authors read and approved the final manuscript.

\section{Ethics approval and consent to participate}

The present study was approved by the Sheba Medical Center Institutional Review Board (Ramat Gan, Israel). All volunteers provided written informed consent.

\section{Patient consent for publication}

Not applicable.

\section{Competing interests}

YM is a shareholder of LifeBiotics Ltd. All the other authors declare that they have no competing interests.

\section{References}

1. de Visser KE, Eichten A and Coussens LM: Paradoxical roles of the immune system during cancer development. Nat Rev Cancer 6: 24-37, 2006.

2. Hughes WT, Armstron D, Bodey GP, Bow EJ, Brown AE, Calandra T, Feld R, Pizzo PA, Rolston KV, Shenep JL and Young LS: 2002 Guidelines for the use of antimicrobial agents in neutropenic patients with cancer. Clin Infet dis 34: 730-751, 2002.

3. Solomayer EF, Feuerer M, Bai L, Umansky V, Beckhove P, Meyberg GC, Bastert G, Schirrmacher V and Diel IJ: Influence of adjuvant hormone therapy and chemotherapy on the immune system analysed in the bone marrow of patients with breast cancer. Clin Cancer Res 9: 174-180, 2003. 
4. Trueman P: Prophylactic G-CSF in patients with early-stage breast cancer: A health economic review. Br J Cancer 101 (Suppl 1): S15-S17, 2009.

5. Zhang M, Liu X, Li J, He L and Tripathy D: Chinese medicinal herbs to treat the side-effects of chemotherapy in breast cancer patients. Cochrane Database Syst Rev 2: CD004921, 2007.

6. Taixiang W, Munro AJ and Guanjian L: Chinese medical herbs for chemotherapy side effects in colorectal cancer patients. Cochrane Database Syst Rev 1: CD004540, 2005.

7. Chen S, Flower A, Ritchie A, Liu J, Molassiotis A, Yu H and Lewith G: Oral Chinese herbal medicine (CHM) as an adjuvant treatment during chemotherapy for non-small cell lung cancer: A systematic review. Lung Cancer 68: 137-145, 2010.

8. Yaal-Hahoshen N, Maimon Y, Siegelmann-Danieli N, Lev-Ari S, Ron IG, Sperber F, Samuels N, Shoham J and Merimsky O: A prospective, controlled study of the botanical compound mixture LCS101 for chemotherapy-induced hematological complications in breast cancer. Oncologist 16: 1197-1202, 2011.

9. Cohen Z, Maimon Y, Yoeli-Lerner M, Yang P, Samuels N and Berger R: Selective anticancer effects and protection from chemotherapy by the botanical compound LCS101: Implications for cancer treatment. Int J Oncol 46: 308-316, 2015.

10. Rachmut IH, Samuels N, Melnick SJ, Ramachandran C, Sharabi Y, Pavlovsky A, Maimon Y and Shoham J: Immunomodulatory effects of the botanical compound LCS101: Implications for cancer treatment. Onco Targets Ther 6: 437-445, 2013.

11. Crawford J, Dale DC and Lyman GH: Chemotherapy-induced neutropenia: Risks, consequences, and new directions for its management. Cancer 100: 228-237, 2004.

12. Bodey GP, Buckley M, Sathe YS and Freireich EJ: Quantitative relationships between circulating leukocytes and infection in patients with acute leukemia. Ann Intern Med 64: 328-340, 1966.
13. Crawford J: Prevention and treatment of chemotherapy-induced neutropenia. Clin Adv Hematol Oncol 11: 514-517, 2013.

14. Wang J, Tong X, Li P, Cao H and Su W: Immuno-enhancement effects of Shenqi Fuzheng Injection on cyclophosphamide-induced immunosuppression in Balb/c mice. J Ethnopharmacol 139: 788-795, 2012.

15. Chen X, Zhang L and Cheung PC: Immunopotentiation and anti-tumor activity of carboxymethylated-sulfated $\beta-(1 \rightarrow 3)$-D-glucan from Poria cocos. Int Immunopharmacol 10: 398-405, 2010.

16. Smiderle FR, Baggio CH, Borato DG, Santana-Filho AP, SassakiGL,Iacomini Mand Van Griensven LJ: Anti-inflammatory properties of the medicinal mushroom Cordyceps militaris might be related to its linear $(1 \rightarrow 3)-\beta-D-$ glucan. PLoS One 9: e110266, 2014.

17. Bone K and Mills S: Principles and Practice of Phytotherapy. 2nd edition. Churchill Livingstone, Edinburgh, 2000.

18. Zhao XZ: Effects of Astragalus membranaceus and Tripterygium hypoglancum on natural killer cell activity of peripheral blood mononuclear in systemic lupus erythematosus. Zhongguo Zhong Xi Yi Jie He Za Zhi 12: 669-671, 645, 1992 (In Chinese).

19. Zhu JS, Halpern GM and Jones K: The scientific rediscovery of a precious ancient Chinese herbal regimen: Cordyceps sinensis: part II. J Altern Complement Med 4: 429-457, 1998.

This work is licensed under a Creative Commons Attribution-NonCommercial-NoDerivatives 4.0 International (CC BY-NC-ND 4.0) License. 